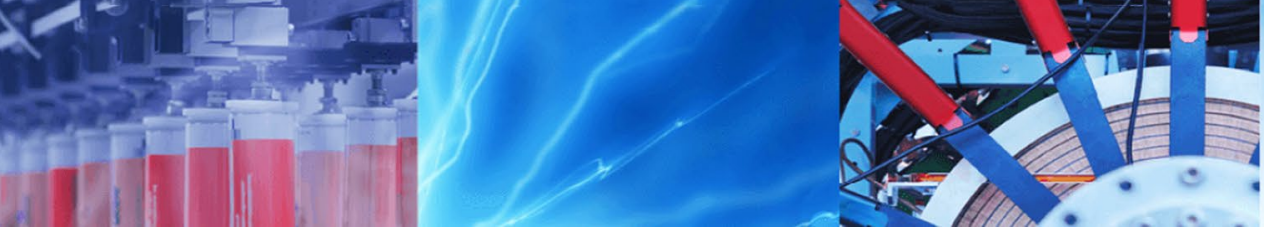

Research Article

\title{
Time complexity analysis of GA-based variants uplink MC-CDMA system
}

\author{
Kashif Iqbal ${ }^{1} \cdot$ Muhammad Adnan Khan $^{1}\left[\right.$ ] $\cdot$ Sagheer Abbas $^{1} \cdot$ Zahid Hasan $^{1}$
}

(c) Springer Nature Switzerland AG 2019

\begin{abstract}
Complexity plays a very significant role in real-time problems. A genetic algorithm (GA)-based multiple input multiple output for an uplink multi-carrier code-division multiple-access (MC-CDMA) receiver is being considered as an important pillar in real-time wireless communication problems. Bit error rate (BER) and minimum mean square error (MMSE) are well-known system performance evolution parameters to estimate the real-time system standards. Sometimes, the multiple solutions give the same BER and MMSE, and then, the complexity is one of the core aspects that will outline the suitable candidate solution. In this article, the proposed drift analysis is being used for the complexity analysis among these GA-based variations. It is observed that the time complexity of the proposed GA-based MC-CDMA with weight relation is less as compared to without weight relation, due to empowering of an exceptional relationship to find out the required solution.
\end{abstract}

Keywords Multi-carrier code-division multiple access (MC-CDMA) · Minimum mean square error (MMSE) · Multi-user detection (MUD) · Bit error rate (BER) · Multiple input multiple output (MIMO) · Genetic algorithm (GA) · Channel estimation (CE)

\section{Introduction}

Day by day the number of users is increasing who are utilizing advance communication technologies. The multicarrier techniques can accommodate the huge number of users in modern communication systems. The multicarrier techniques such as multi-carrier direct-sequence code-division multiple access (MC-DS-CDMA), orthogonal frequency-division multiple access (OFDMA) and multicarrier code-division multiple access (MC-CDMA) are wellknown techniques which can accommodate a large number of users. High data rates demands are fulfilled using MIMO systems with the MC-CDMA system [1, 2]. In a MIMO system, multiple transmission and receive antennas are used to achieve the high data rate demand of advanced wireless communication systems [3-5].

One of the principal causes of the poor performance of MIMO-based MC-CDMA is multi-user interference (MUI). There are solutions to such problems by implementing the uplink receiver adaptively. One of the adaptive uplink receiver solutions is the LMS (least mean square) receiver proposed by Seo et al. [6] and Mezghani and SwindleHurst [7]. Its traditional version of MC-CDMA receiver is introduced in [8,9] as well. In [10], Asad et al. applied hybrid computational intelligence techniques for blind-, semiblind- and training-based joint channel and data estimation model. The proposed techniques show attractive

$\triangle$ Muhammad Adnan Khan, madnankhan@ncbae.edu.pk; Kashif Iqbal, Kashif.lqbal.shah@gmail.com; Sagheer Abbas, dr.sagheer@ ncbae.edu.pk; Zahid Hasan, zahidhassan@ncbae.edu.pk | 'School of Computer Science, National College of Business Administration and Economics, Lahore, Pakistan.

SN Applied Sciences (2019) 1:953 | https://doi.org/10.1007/s42452-019-0984-4

Received: 21 May 2019 / Accepted: 24 July 2019 / Published online: 1 August 2019 
results regarding BER, MMCE, MMSE as compared to conventional opposite learning mutant PSO techniques.

So, different methods are used for the adaptive uplink receiver. In some cases, these proposed multiple receiver's algorithms such as genetic algorithm (GA), least mean square (LMS), recursive least square (RLS) and differential evolutionary (DE) are given the same bit error rate (BER) or minimum mean square error (MMSE) as proposed by Seo et al. [6], Umair et al. [11], Khan et al. [8, 9] and Ali et al. [12, 13] after fixing iterative solutions. The time and space complexity was discussed by Tariq Zaid et al. [14] and Asif et al. [15] using the differential evolutionary (DE)-based CE-UD and the PSO-based MIMO system for channel estimation and multi-user detection.

The time complexity plays a vital role in the evaluation of the effect of relation. There are different complexity analysis systems such as drift analysis, negative analysis and population-based analysis.

In this article, we are using a population-based drift analysis algorithm to evaluate the performance of the GAbased MC-CDMA system with two variants.

The objective of this paper is to optimize the time and space complexity algorithm of the system model using computational intelligence techniques. The genetic algorithm is under discussion with the proposed variation with and without weight relation. The rest of the paper is structured as follows: Sect. 2 gives an overview of the system model taken from the background international literature. Section 3 presents the time complexity analysis of GAbased algorithm with weight relation and without weight relation, while Sect. 4 provides the simulation results of the time complexity of GA-based MC-CDMA system with and without weight relation regarding the simulation analysis results of NoCs, Ls and the number of users. The conclusion is given in the last section that explains that the time and space complexity is directly proportional to the number of users, multiple channel paths and the number of cycles.

\section{System model}

In this article, two transmit antennas and one receiving antenna are used with the MC-CDMA system. The received signal vector for two consecutive symbols is given by Seo et al. [6], Khan et al. [8, 9] and Ali et al. [12, 13] as follows:

$$
\begin{aligned}
& \mathbf{R}(j)=\left[\begin{array}{ll}
\mathbf{r}^{\top}(2 j-1) & \mathbf{r}^{H}(2 j)
\end{array}\right]^{\top} \\
& \mathbf{R}(j)=\sum_{i=1}^{U}\left\{\mathbf{S}_{i, 1} d_{i}(2 j-1)+\mathbf{S}_{i, 2} d_{i}(2 j)\right\}+\mathbf{N}(j)
\end{aligned}
$$

In the above equation, $\mathbf{S}, d$ and $\mathbf{N}$ represent the channel response, transmitted symbols and additive white Gaussian noise, respectively.

The minimum mean square error (MMSE) of the uplink receiver is attained by minimizing the equation proposed in [6]

$W\left[\mathbf{c}_{o, 1}, \mathbf{c}_{o, 2}\right]=\arg W\left(\mathbf{c}_{1}, \mathbf{c}_{2}\right)$

$W\left[\mathbf{c}_{o, 1}, \mathbf{c}_{o, 2}\right]=\left\{W_{1}\left(\mathbf{c}_{1}\right)+W_{2}\left(\mathbf{c}_{2}\right)\right\}$

The following relationship is calculated in Seo et al. [6], Khan et al. [8, 9] and Umair et al. [11].

$\mathbf{c}_{1,2}=\mathbf{c}_{2,3}^{*}$ and $\mathbf{c}_{1,4}=-\mathbf{c}_{1,1}^{*}$

The improved cast function $W$ can be written as:

$W_{z}=W_{z 1}\left(\mathbf{c}_{\mathrm{a}^{\prime}} \mathbf{c}_{\mathrm{b}}\right)+W_{z 2}\left(\mathbf{c}_{\mathrm{a}}, \mathbf{c}_{\mathrm{b}}\right)$

where

$W_{z 1}\left(\mathbf{c}_{\boldsymbol{a}}, \mathbf{c}_{\mathbf{b}}\right)=E\left[\left|\mathbf{c}_{a}^{H} \mathbf{r}(2 j-1)+\mathbf{c}_{\mathrm{b}}^{\top} \mathbf{r}^{*}(2 j)-d_{1}(2 j-1)\right|^{2}\right]$

and

$W_{z 2}\left(\mathbf{c}_{\boldsymbol{a}}, \mathbf{c}_{\mathrm{b}}\right)=E\left[\left|\mathbf{c}_{b}^{H} \mathbf{r}(2 j-1)+\mathbf{c}_{\mathrm{a}}^{\top} \mathbf{r}^{*}(2 j)-d_{1}(2 j-1)\right|^{2}\right]$,

GA is very frequently used in nonlinear problems by the optimization algorithm efficiently. The candidate solution in GA is presented as a chromosome. Chromosome naturally includes breeding and mutation behaviour proposed by [16] and Soltani et al. [17]. Same as this phenomenon, the new population is produced by reproduction and mutation.

The GA is used for minimizing the problem given in Eqs. (3) and (4). The first one is the primary cost function, and the second is the improved cost function. The GA algorithm is given in Table 1. The detailed discussion of all its steps is provided in the table.

\section{Complexity of GA-based algorithm with weight relation (WWR)}

The algorithm is defined in a step-by-step procedure to solve a problem using a computer. The complexity determines how efficient an algorithm is regarding time and space utilization. In other words, the complexity shows how fast or how slow an algorithm works. The functional complexity is defined as $T(n)$ where $T$ is the time and $n$ is the size of the input. After a fix number of cycles, if complicated algorithms give same bit error rate and minimum mean square error, then the complexity is one of the factors which will determine which algorithm is the best as proposed by Abdelkader et al. [18]. 
Table 1 Genetic algorithm

\begin{tabular}{ll}
\hline S. no. & Steps \\
\hline 1. & Start \\
2. & Initialization of weights $c_{u}$ \\
3. & Calculate the fitness function using the cost function given in Eq. (3) \\
4. & Sort the weights in ascending order as per fitness values \\
5. & Select the best parents \\
6. & Generate the children using the crossover \\
7. & (the crossover ration is $1 / 2$ and $1 / 2$ of Parent. No. 1 and Parent. No. 2) \\
8. & The mutation process is applied \\
9. & Calculate the fitness \\
10. & If (number of cycles or required BER achieved) go to step 10 \\
\hline
\end{tabular}

\subsection{The complexity of GA-based algorithm WWR is given by}

Step 1 Data population initialization

for $\mathrm{i} \rightarrow 1: \mathrm{U}$

for $\mathrm{j} \rightarrow 1: \mathrm{Ls}$

$\mathrm{P}_{\mathrm{C}}(\mathrm{i}, \mathrm{j})=\operatorname{rand}()$

end

end

Complexity of initialization of data population:

$$
\begin{aligned}
& T_{2}=\sum_{i=1}^{U} \sum_{j=1}^{\mathrm{Ls}} 1 \\
& T_{2}=\sum_{i=1}^{U} \mathrm{Ls}=\mathrm{Ls} \sum_{i=1}^{U} 1=\mathrm{Ls} U
\end{aligned}
$$

Step 2 Complexity of user initialization

$$
T_{3}=U
$$

Step 3

In step $3 \& 4$ population fitness is calculated and sorted using merge sort. The complexity to calculate the eligibility and data population is $C$. The complexity of merge sort is given by:

Merge-sort (arr B, int q, int w)

$$
\text { if }(\mathrm{q}<\mathrm{m})
$$

then

$$
\begin{aligned}
& \mathrm{m} \leftarrow(\mathrm{q}+\mathrm{w}) / 2 \\
& \operatorname{Merge-sort}(\mathrm{B}, \mathrm{q}, \mathrm{m}) \\
& \operatorname{Merge-sort}(\mathrm{B}, \mathrm{m}+1, \mathrm{w}) \\
& \operatorname{Merge-sort}(\mathrm{B}, \mathrm{q}, \mathrm{m}, \mathrm{w})
\end{aligned}
$$

Recursive function for merge sort is:

$T(U)= \begin{cases}1 & \text { if } U=1 \\ 2 T\left(\frac{U}{2}\right)+U & \text { otherwise }\end{cases}$

Substituting $U=U / 2$ in Eq. (i), we get:

$T\left(\frac{U}{2}\right)=2 T\left(\frac{\frac{U}{2}}{2}\right)+\frac{U}{2}$

$T\left(\frac{U}{2}\right)=2 T\left(\frac{U}{4}\right)+U / 2$

Now, substituting the value of $T(U / 2)$ in Eq. (i), we get: 


$$
\begin{aligned}
T(U) & =2\left[2 T\left(\frac{U}{4}\right)+\frac{U}{2}\right]+U \\
& =4 T\left(\frac{U}{4}\right)+2 U \\
T(U) & =2^{2} T\left(\frac{U}{2^{2}}\right)+2 U
\end{aligned}
$$

Now, substituting $U=U / 4$ in Eq. (i), we get:

$$
\begin{aligned}
T(U) & =2 T\left(\frac{U}{2}\right)+U \\
& =2 T\left(\frac{\frac{U}{4}}{2}\right)+\frac{U}{4} \\
& =2 T\left(\frac{U}{8}\right)+U / 4
\end{aligned}
$$

Substituting the value of $T(U / 4)$ in Eq. (ii), we get:

$$
\begin{aligned}
T(U) & =4\left[2 T\left(\frac{U}{8}\right)+\frac{U}{4}\right]+2 U \\
& =8 T\left(\frac{U}{8}\right)+3 U \\
T(U) & =2^{3} T\left(\frac{U}{2^{3}}\right)+3 U
\end{aligned}
$$

Now, substituting the value of $U=U / 8$ in Eq. (i), we get:

$$
\begin{aligned}
T\left(\frac{U}{8}\right) & =2 T\left(\frac{\frac{U}{8}}{2}\right)+\frac{U}{8} \\
& =2 T\left(\frac{U}{16}\right)+\frac{U}{8}
\end{aligned}
$$

Now, substituting the value of $U / 8$ in Eq. (iii), we get:

$$
\begin{aligned}
T(U) & =8\left[2 T\left(\frac{U}{16}\right)+\frac{U}{8}\right]+3 U \\
& =16 T\left(\frac{U}{16}\right)+4 U \\
T(U) & =2^{4} T\left(\frac{U}{2^{4}}\right)+4 U \\
T(U) & =2^{k} T\left(\frac{U}{2^{k}}\right)+k U
\end{aligned}
$$

From Eq. (v) after substitution, we get:

By taking $2^{k}=U$ and putting $k=4$ in equation $(v)$ we get

Taking log on both sides:

$\log U=\log 2^{k}$ $\log U=k$

or

$k=\log U$

Substituting the values of $U$ and $k$ in Eq. (v), we get:

$$
\begin{aligned}
T(U) & =U T\left(\frac{U}{U}\right)+k U \\
& =U T\left(\frac{U}{U}\right)+\log U * U \\
T(U) & =U T(1)+U \log U
\end{aligned}
$$

From the recursive equation, it can be seen that $T(1)=1$. So, the above equation becomes:

$T(U)=U+U \log U$

So, the complexity of merge sort is $U \log U$.

The complexity of step 3 for $T_{4}$ is given by:

$T_{4}=\mathrm{Ls}+U \log U$

And the number of carriers in Step 5 is:

$T_{5}=C$

Step 6

$r_{\mathrm{c}}$ used here is for crossover $0 \leq r_{\mathrm{c}} \leq 1$

$$
T_{6}=U+U_{r_{c}}
$$

Step 7

$r_{\mathrm{m}}$ is used here for the mutation process

$$
T_{7}=\left(U+U_{r_{c}}\right) r_{m}
$$

\section{Step 8}

$$
T_{8}=\left(U+U_{r_{c}}\right)
$$

Total complexity of GA-based algorithm is:

$$
\begin{aligned}
T= & T_{2}+\left(T_{3}+T_{4}+T_{5}+T_{6}+T_{7}+T_{8}\right) N c \\
T= & L s U+\left[U+\left(U+L s \log _{10} U\right)+C+\left(U+U r_{c}\right)\right. \\
& \left.+\left(U+U r_{c}\right) r_{m}+U+U r_{c}\right] N c \\
T= & L s U+\left[U+U+L s \log _{10} U+U r_{\mathrm{c}}+U r_{\mathrm{m}}+U r_{\mathrm{c}} r_{\mathrm{m}}\right. \\
& \left.+U+U r_{\mathrm{c}}+C\right] N c \\
T= & \mathrm{Ls} U+\left[4 U+U\left(2 r_{\mathrm{c}}+r_{\mathrm{m}}\left(1+r_{\mathrm{c}}\right)+\mathrm{Ls} \log _{10} U\right] N c\right.
\end{aligned}
$$

\subsection{The complexity of GA-based algorithm without weight relation}

The functional complexity is defined as $T_{\text {wow }}(n)$ where $T_{\text {wow }}$ is the time and $n$ is the size of the input. After a fix number of cycles, if multiple algorithms gives the same bit error rate 
Fig. 1 Number of cycles versus time complexity with different number of users

\section{$\mathrm{Ls}=16, \mathrm{rc}=.5, \mathrm{rm}=.5$}

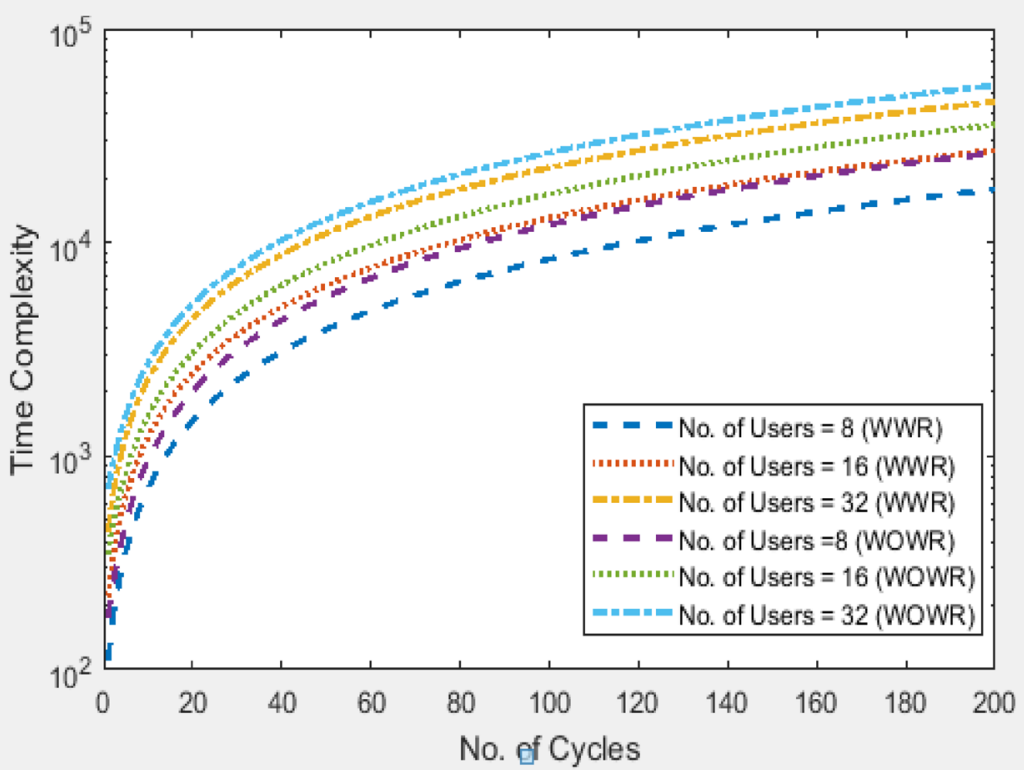

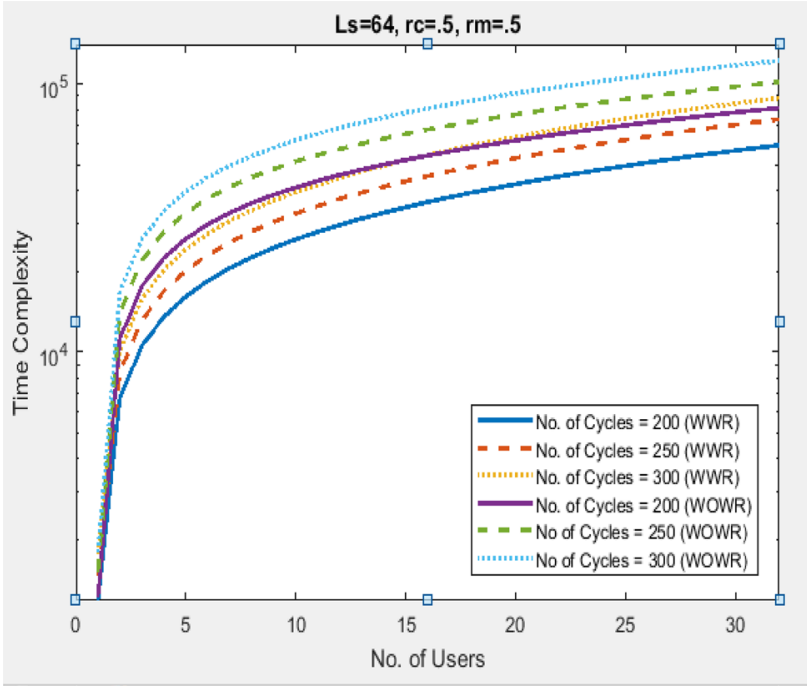

Fig. 2 Number of users versus time complexity with different number of cycles

and minimum mean square error, then the complexity is one of the factors which will determine which algorithm is best $[6,8,9,11-13]$.

Total complexity of GA-based algorithm is:

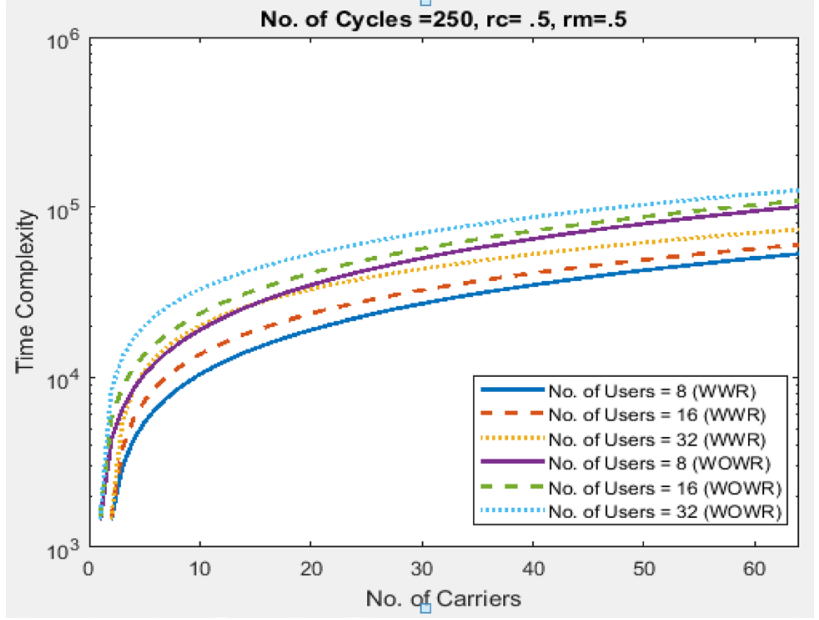

Fig. 3 Number of carriers versus time complexity with different number of users

\section{Simulation results and discussion}

The complexity of the GA-based uplink MC-CDMA is implemented with sub-carriers (Ls), users $(U)$ and the number of cycles (NoCs). The number of sub-carriers is equal to the length of spreading codes.

$$
\begin{aligned}
& T_{\text {wow }}=T_{2}+\left(T_{3}+T_{4}+T_{5}+T_{6}+T_{7}+T_{8}\right) N c \\
& T_{\text {wow }}=\frac{1}{2} \mathrm{Ls} U+\left[U+\left(U+\frac{1}{2} \operatorname{Ls} \log _{10} U\right)+C+\left(U+U r_{\mathrm{c}}\right)+\left(U+U r_{\mathrm{c}}\right) r_{\mathrm{m}}+U+U r_{\mathrm{c}}\right] N c \\
& T_{\text {wow }}=\frac{1}{2} \mathrm{Ls} U+\left[U+U+\frac{1}{2} \operatorname{Ls} \log _{10} U+U r_{\mathrm{c}}+U r_{\mathrm{m}}+U r_{\mathrm{c}} r_{\mathrm{m}}+U+U r_{\mathrm{c}}+C\right] N c \\
& T_{\text {wow }}=\frac{1}{2} \mathrm{Ls} U+\left[4 U+U\left(2 r_{\mathrm{c}}+r_{\mathrm{m}}\left(1+r_{\mathrm{c}}\right)+\frac{1}{2} \mathrm{Ls} \log _{10} U\right] N c\right.
\end{aligned}
$$




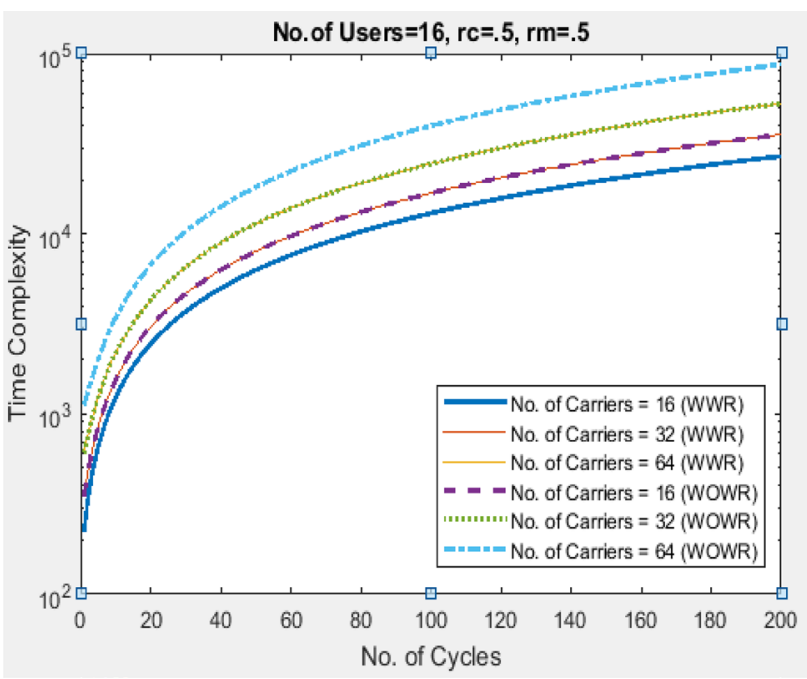

Fig. 4 Number of cycles versus time complexity with different number of carriers

Figures 1, 2, 3 and 4 show the simulation results of the time complexity for GA-based MC-CDMA system without weight relation and with weight relation regarding $\mathrm{NoCs}$, Ls and $U$.

Figure 1 shows the time complexity of GA-based MCCDMA with and without weight relation with respect to the different number of cycles and users. The top curves, middle curves and bottom curves show the time complexity when the number of users is 8,16 and 32 , respectively. It is shown in the graph that when the number of users and the number of carriers are increased, the time complexity is also increased. It further indicates that the time complexity of the proposed GA-based MC-CDMA with weight relation is less as compared to without weight relation algorithm-based solution.

Figure 2 shows the time complexity of the proposed algorithm versus the number of cycles (NoCs) with the different number of users $(U)$. The top curves, middle curves and bottom curves show the time complexity when the number of cycles is 200, 250 and 300 , respectively. It clearly shows that when the number of cycles and the number of carriers are increased, then the time complexity is also increased, which shows the positive correlation between two variables. It further indicates that the time complexity of the proposed GA-based MC-CDMA with weight relation is less as compared to without weight relation algorithmbased solution.

Figure 3 shows the time complexity of the proposed algorithm versus the number of carriers (Ls) with the different number of users $(U)$. The top curves, middle curves and bottom curves show the time complexity when the different number of users (Us) is 8,16 and 32 , respectively. It clearly shows that when the number of users and the number of cycles are increased, then the time complexity is also increased, which shows the positive correlation between two variables. It further indicates that the time complexity of the proposed GA-based MC-CDMA with weight relation is less as compared to without weight relation algorithm-based solution.
Table 2 Time complexity of GA-based variants MC-CDMA system

\begin{tabular}{|c|c|c|c|c|c|}
\hline \multirow[t]{2}{*}{ Parameters } & \multicolumn{2}{|c|}{ Number of operations (NoOs) } & \multicolumn{2}{|c|}{$\begin{array}{l}\text { Time complexity (Intel core } 2 \\
\text { duo }=0.0004 \text { ( } \mu \text { s/ins) }\end{array}$} & \multirow{2}{*}{$\begin{array}{l}\text { Time saving } \\
T(\mathrm{~S} 2)-T(\mathrm{~S} 1)\end{array}$} \\
\hline & $\begin{array}{l}\text { GA with weight } \\
\text { relation (S1) }\end{array}$ & $\begin{array}{l}\text { GA without } \\
\text { weight relation } \\
\text { (S2) }\end{array}$ & $\begin{array}{l}\text { GA with weight } \\
\text { relation } T(\mathrm{~S} 1)\end{array}$ & $\begin{array}{l}\text { GA without } \\
\text { weight relation } \\
T(\mathrm{~S} 2)\end{array}$ & \\
\hline \multicolumn{6}{|c|}{ Number of users with $(\mathrm{NoCs}=200)$} \\
\hline$U=8$ & 17,742 & 26,283 & 8 & 11 & 3 \\
\hline$U=16$ & 27,006 & 35,611 & 11 & 15 & 4 \\
\hline$U=32$ & 45,534 & 54,267 & 19 & 22 & 3 \\
\hline$U=512$ & 601,374 & 613,947 & 241 & 246 & 5 \\
\hline \multicolumn{6}{|c|}{ Number of carriers with $(\mathrm{NoCs}=200)$} \\
\hline $\mathrm{Ls}=32$ & 27,006 & 35,611 & 11 & 15 & 4 \\
\hline $\mathrm{Ls}=64$ & 35,611 & 52,822 & 15 & 22 & 7 \\
\hline $\mathrm{Ls}=128$ & 52,822 & 87,243 & 22 & 35 & 13 \\
\hline $\mathrm{Ls}=512$ & 293,770 & 569,140 & 118 & 238 & 120 \\
\hline \multicolumn{6}{|c|}{ Number of cycles with $(U=64)$} \\
\hline $\mathrm{NoCs}=200$ & 59,045 & 81,257 & 24 & 33 & 9 \\
\hline $\mathrm{NoCs}=250$ & 73,790 & 101,548 & 30 & 41 & 11 \\
\hline $\mathrm{NoCs}=300$ & 88,536 & 121,839 & 36 & 49 & 13 \\
\hline $\mathrm{NoCs}=500$ & 147,516 & 203,000 & 60 & 82 & 22 \\
\hline
\end{tabular}




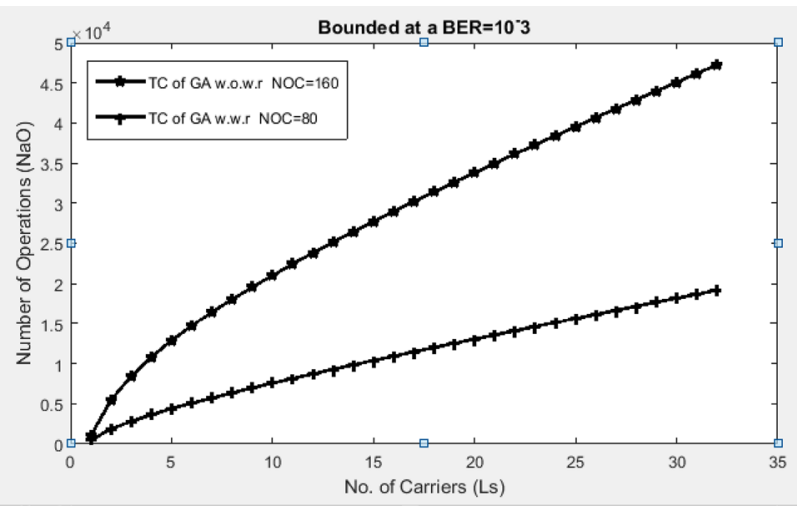

Fig. 5 Number of carriers (Ls) and number of operations (NoOs) with varying number of cycles

Figure 4 shows the time complexity of the proposed algorithm versus the number of cycles (NoCs) with the different number of carriers ( $L s)$. The top curves, middle curves and bottom curves show the time complexity when the different number of carriers (Ls) is 16, 32 and 64 , respectively. It clearly shows that when the number of users and the number of carriers are increased, then the time complexity is also increased, which shows the positive correlation between two variables. It further indicates that the time complexity of the proposed GA-based MCCDMA with weight relation is less as compared to without weight relation algorithm-based solution.

Table 2 shows the time complexity of GA-based variants MC-CDMA system with respect to the different number of cycles, sub-carriers and users. It is observed that if some sequences are fixed and some users are varied approximately, both GA-based variants give the same time complexity approximation. But when some carriers are changed, e.g. $L s=512, G A$ with weight relation solves the problem in $118 \mathrm{~ns}$, but GA without weight relation takes $238 \mathrm{~ns}$. It means that GA with weight connection is two times faster as compared to GA without a weight relation-based solution. Similarly, GA with weight relation saves 22 ns when users are fixed $(U=64)$, and the number of cycles (NoCs) is changed. In this case, when the number of periods is $500 \mathrm{~ns}, \mathrm{GA}$ with weight relation saves $22 \mathrm{~ns}$.

Figure 5 shows the number of carriers (Ls) versus the number of operations (NoOs) at bounded bit error rate (BER) at $10^{-3}$ of the proposed GA-based algorithmic scheme. The top curve shows that GA (WOWR) required 160 cycles, and GA (WWR) required 80 cycles to achieve $10^{-3} \mathrm{BER}$. It is observed that when the number of carriers is increased, the number of operations (NoOs) is also increased. It further shows that GA WWR-based solution required less number of operations as compared to GA WOWR-based solution. It means that GA (WWR) time complexity is less as compared to GA (WOWR)-based solution.

Table 3 shows the time complexity of GA-based variants MC-CDMA system with bounded bit error rate (BER) at $10^{-3}$. It is observed that $\mathrm{GA}$ with weight relation has achieved required BER in $8 \mathrm{~ns}$, but GA-based without weight relation variant takes $19 \mathrm{~ns}$. GA with weight relation solution is approximately two times faster as compared to GA without weight relation-based solution.

\section{Conclusion}

The objective of this work is the complexity analysis of GAbased variants uplink receiver MC-CDMA system methods. Initially, evolutionary techniques are applied for uplink MC-CDMA systems to improve the time and space complexity of channel and its quality. In the adaptive genetic algorithm (AGA) in communication for time and capacity improvement, the number of users is increased and high data rate is needed. The MIMO technology is used to improve the function of the channel, data rate and service quality for communication systems. In this paper, the time complexity of GA-based variants uplink receiver MC-CDMA system is computed. The time and memory complexity is directly proportional to the number of users, multiple channel paths and the number of cycles. It is also observed that in the case of bounded BER and number of carriers, $G A$ with weight relation-based solution is two times faster as compared to GA without weight relation-based MCCDMA system. It is observed that when channel paths,
Table 3 Time complexity of GA-based variants MC-CDMA system bounded at a $\mathrm{BER}=10^{-3}$

\begin{tabular}{lccccc}
\hline Algorithm & U/Ls & BER & $\begin{array}{l}\text { NoCs required } \\
\text { achieving 10 } \\
\text { MMSE }\end{array}$ & NoOs & $\begin{array}{l}\text { Time complexity (Intel } \\
\text { core 2 duo }=0.0004(\mu \mathrm{s} / \\
\text { ins) }\end{array}$ \\
\hline GA with weight relation (S1) & $16 / 32$ & $10^{-3}$ & 80 & 19,205 & 8 \\
GA without weight relation (S2) & $16 / 32$ & $10^{-3}$ & 160 & 47,249 & 19 \\
Time saving $T(\mathrm{~S} 2)-T(\mathrm{~S} 1)$ & & & & 11 \\
\hline
\end{tabular}


number of users and number of cycles are increased, the complexity is also increased.

\section{Compliance with ethical standards}

Conflict of interest The authors declared that they have no conflict of interest.

\section{References}

1. Raut PW, Badjate SL (2013) MIMO-future wireless communication. Int J Innov Technol Explor Eng 2(5):102-106

2. Pirak C, Wang ZJ, Liu KR, Jitapunkul S (2006) Optimum power allocation for maximum-likelihood channel estimation in spacetime coded MIMO systems. In: Acoustics, speech and signal processing, 2006. 2006 IEEE international conference on ICASSP 2006 proceedings, vol 4. IEEE

3. Akhtman J, Hanzo L (2006) Reduced-complexity maximumlikelihood detection in multiple-antenna-aided multicarrier systems, 5th Int. Workshop Multi-Carrier Spread Spectrum Communications, Germany, pp 14-16

4. Abuthinien M, Chen S, Wolfgang A, Hanzo L (2007) Joint maximum likelihood channel estimation and data detection for MIMO systems. In: IEEE international conference on communications, 2007. ICC'07, pp 5354-5358. IEEE

5. Seyman MN, Taspinar N (2013) Symbol detection using the differential evolution algorithm in MIMO-OFDM systems. Turk J Electr Eng Comput Sci 21(2):373-380

6. Seo B, Ahn WG, Jeong C, Kim HM (2010) Fast convergent LMS adaptive receiver for MC-CDMA systems with space-time block coding. IEEE Commun Lett 14(8):737-739

7. Mezghani A, Swindlehurst $L$ (2017) Blind estimation of sparse multi-user massive MIMO channels. In: WSA 2017; 21st International ITG Workshop on Smart Antennas. VDE, pp 1-5

8. Khan MA, Umair M, Choudhry MAS (2015) GA based adaptive receiver for MC-CDMA system. Turk J Electr Eng Comput Sci 23(Sup. 1):2267-2277

9. Khan MA, Umair M, Choudhry MAS (2015) Island differential evolution based adaptive receiver for MC-CDMA system. In: IEEE international conference on information and communication technologies (ICICT), pp 1-6
10. AsadUllah M, Khan MA, Abbas S, Athar A, Raza SS, Ahmad G (2018) Blind channel and data estimation using fuzzy logicempowered opposite learning-based mutant particle swarm optimization. Comput Intell Neurosci

11. Umair M, Khan MA, Saleem MA (2015) Island genetic algorithm based MUD for MC-CDMA System. In: 6th IEEE international conference on information and communication technologies, electronic ISBN: 978-1-4673-8907-5, Electronic ISBN: 978-14673-8907-5, https://doi.org/10.1109/ICICT.2015.7469588, INSPEC Accession Number: 16004107, pp 7-12

12. Ali MN, Amir M, Khan MA (2016) Heuristic computation based adaptive receiver for MC-CDMA. In: 3rd international conference on engineering and emerging technologies (ICEET-2016) conference proceedings ISSN: 2409-2983

13. Ali N, Khan MA, Adeel M, Amir M (2016) Genetic algorithm based adaptive receiver for MC-CDMA system with variation in mutation operator. Int J Comput Sci Inf Secur 14(9):215-222

14. Tariq ZB, Khan MA, Abbas S, Fatima A (2019) Complexity analysis of DE based CE-UD MIMO system. In: 2019 2nd international conference on computing, mathematics and engineering technologies (iCoMET), pp 1-5. IEEE

15. Asif M, Khan MA, Abbas S, Saleem M (2019) Analysis of space and time complexity with PSO based synchronous MC-CDMA system. In: 2019 2nd international conference on computing, mathematics and engineering technologies (iCoMET), pp 10-15. IEEE

16. Yuen SY, Chow CK (2009) A genetic algorithm that adaptively mutates and never revisits. IEEE Trans Evol Comput 13(2):454-472

17. Soltani MD, Aghaeinia H, Alimadadi M (2014) A low complexity suboptimal energy-based detection method for SISO/MIMO channels. Wirel Pers Commun 77(4):2857-2869

18. Abdelkader T, Mokhtar K, Abdelaziz O (2014) New space time coding for joint blind channel estimation and data detection through time varying MIMO channels. Int J Comput Sci Issues (IJCSI) 11(5):33

Publisher's Note Springer Nature remains neutral with regard to jurisdictional claims in published maps and institutional affiliations. 